PROCEEDINGS OF THE

AMERICAN MATHEMATICAL SOCIETY

Volume 135, Number 1, January 2007, Pages 13-20

S 0002-9939(06)08443-7

Article electronically published on June 19, 2006

\title{
CONVOLUTION CONGRUENCES FOR THE PARTITION FUNCTION
}

\author{
SHARON ANNE GARTHWAITE
}

(Communicated by Ken Ono)

\begin{abstract}
Ahlgren and Boylan recently proved the uniqueness of the Ramanujan congruences for the primes 5,7 , and 11 by using the modularity of a certain partition function. Here we use their result to find universal congruences, of a different type, which hold for the partition function modulo all primes $\ell \geq 5$.
\end{abstract}

\section{Introduction AND STATEMENT OF RESUlts}

Let $p(n)$ denote the number of partitions of the positive integer $n$, that is, the number of representations of $n$ as the sum of a sequence of non-increasing positive integers. For more on partitions see And98] or AO01. For convenience, we let $p(0)=1$, and $p(\alpha)=0$ if $\alpha<0$. Euler introduced the generating function

$$
\sum_{n \geq 0} p(n) q^{n}=\prod_{n \geq 1} \frac{1}{1-q^{n}} .
$$

If we denote the pentagonal numbers

$$
\omega(k):=\frac{1}{2} k(3 k+1), \quad k \in \mathbb{Z},
$$

then we have

$$
\sum_{k \in \mathbb{Z}}(-1)^{k} q^{\omega(k)} \cdot \sum_{n \geq 0} p(n) q^{n}=1 .
$$

This gives rise to the recursive formula

$$
p(n)=\sum_{k \in \mathbb{Z}-\{0\}}(-1)^{k+1} p(n-\omega(k)),
$$

which holds for all positive integers $n$. While this formula is useful for quickly computing values of $p(n)$, it has not offered much information about the arithmetic of the partition function.

The famous Ramanujan congruences

$$
\begin{aligned}
p(5 n+4) & \equiv 0 \quad(\bmod 5), \\
p(7 n+5) & \equiv 0 \quad(\bmod 7), \\
p(11 n+6) & \equiv 0 \quad(\bmod 11),
\end{aligned}
$$

Received by the editors July 5, 2005 and, in revised form, July 25, 2005.

2000 Mathematics Subject Classification. Primary 11P83; Secondary 11F11.

This research was supported by the University of Wisconsin Madison NSF VIGRE program.

(C)2006 American Mathematical Society Reverts to public domain 28 years from publication 
may be reformulated in the following peculiar way. For primes $\ell \geq 5$, define

$$
\delta_{\ell}:=\frac{\ell^{2}-1}{24}
$$

and let

$$
\mathcal{P}_{\ell}(z):=\sum_{k \in \mathbb{Z}}(-1)^{k} q^{\ell \omega(k)} \sum_{n \geq 0} p\left(\ell n-\delta_{\ell}\right) q^{n} .
$$

The Ramanujan congruences are equivalent to the assertion that $\mathcal{P}_{\ell}(z) \equiv 0(\bmod \ell)$ for $\ell \in\{5,7,11\}$. For example, we have

$$
\begin{aligned}
\mathcal{P}_{5}(z) & =5 q+30 q^{2}+135 q^{3}+490 q^{4}+1575 q^{5}+\cdots \equiv 0 \quad(\bmod 5) \\
\mathcal{P}_{7}(z) & =7 q+77 q^{2}+490 q^{3}+2436 q^{4}+10143 q^{5}+\cdots \equiv 0 \quad(\bmod 7) \\
\mathcal{P}_{11}(z) & =11 q+297 q^{2}+3718 q^{3}+31185 q^{4}+204226 q^{5}+\cdots \equiv 0 \quad(\bmod 11)
\end{aligned}
$$

Ahlgren and Boylan AB03, have proven Ramanujan's famous conjecture that congruences of the form $p(\ell n-\beta) \equiv 0(\bmod \ell)$ exist only for primes 5,7 , and 11 . Therefore, it is natural to ask whether some refinement of (1.3) continues to hold for all primes $\ell \geq 5$. Here we show that this is indeed the case.

To make this precise, let $M_{k}$ denote the space of weight $k$ holomorphic modular forms on $\mathrm{SL}_{2}(\mathbb{Z})$. For more on modular forms see Kob93. We shall denote a modular form $f(z) \in M_{k}$ by its Fourier expansion

$$
f(z):=\sum_{n=0}^{\infty} a_{f}(n) q^{n}, \quad q:=e^{2 \pi i z} .
$$

For each prime $\ell$ we have the $U(\ell)$-operator defined by

$$
f(z) \mid U(\ell)=\sum_{n=0}^{\infty} a_{f}(\ell n) q^{n} .
$$

Furthermore, for even $k \geq 4$, let $E_{k}(z) \in M_{k}$ denote the normalized Eisenstein series of weight $k$,

$$
E_{k}(z):=1-\frac{2 k}{B_{k}} \sum_{n=1}^{\infty} \sigma_{k-1}(n) q^{n},
$$

where the rational numbers $B_{k}$ are the Bernoulli numbers defined by

$$
\sum_{n=0}^{\infty} B_{n} \cdot \frac{t^{n}}{n !}=\frac{t}{e^{t}-1}=1-\frac{1}{2} t+\frac{1}{12} t^{2}+\cdots
$$

and

$$
\sigma_{k-1}(n)=\sum_{1 \leq d \mid n} d^{k-1} .
$$

Our refinement of (1.3) is the following.

Theorem 1.1. Suppose $\ell \geq 5$ is prime and $m \in\{4,6,8,10,14\}$. If $\mathcal{P}_{\ell}^{(m)}(z):=$ $E_{m}(z) P_{\ell}(z)$, then

$$
\mathcal{P}_{\ell}^{(m)}(z) \mid U(\ell) \equiv 0 \quad(\bmod \ell)
$$


Remark. Note that $E_{4}(z)=1+240 \sum_{n=1}^{\infty} \sigma_{3}(n) q^{n} \equiv 1(\bmod 5)$. Similarly, $E_{6}(z) \equiv$ $1(\bmod 7)$ and $E_{10}(z) \equiv 1(\bmod 11)$. While this does not yield the full Ramanujan congruences, it does show

$$
p\left(\ell^{2} n-\delta_{\ell}\right) \equiv 0 \quad(\bmod \ell) \quad \text { for } \ell=5,7,11 .
$$

Example. If $m=4$ and $\ell=13$, then

$$
\mathcal{P}_{13}^{(4)}(z)=11 q+3130 q^{2}+149709 q^{3}+3225214 q^{4}+43626740 q^{5}+\cdots,
$$

$\mathcal{P}_{13}^{(4)}(z) \mid U(13)=64580467571997 q+166693095587328996218 q^{2}+\cdots \equiv 0(\bmod 13)$.

If we let $b(4):=240, b(6):=-540, b(8):=480, b(10):=-264, b(14):=-24$, then Theorem 1.1 implies the following recursive congruence.

Theorem 1.2. For each prime $\ell \geq 5$ and each $m \in\{4,6,8,10,14\}$ we have

$$
\begin{aligned}
p\left(\ell^{2} N-\delta_{\ell}\right) \equiv & \sum_{k \in \mathbb{Z}-\{0\}}(-1)^{k+1} p\left(\ell^{2}(N-\omega(k))-\delta_{\ell}\right) \\
& +b(m) \sum_{\substack{n \geq 1 \\
k \in \mathbb{Z}}} \sigma_{m-1}(n)(-1)^{k+1} p\left(\ell^{2}(N-\omega(k)-n / \ell)-\delta_{\ell}\right) \quad(\bmod \ell)
\end{aligned}
$$

for all positive integers $N$.

Theorem 1.1 is a consequence of a generic theorem for cusp forms on $\mathrm{SL}_{2}(\mathbb{Z})$ with integer coefficients. Let $S_{k}$ denote the space of cusp forms of weight $k$, that is, the subspace of modular forms $f(z) \in M_{k}$ such that $a_{f}(0)=0$. For example,

$$
\Delta(z):=q \prod_{n=1}^{\infty}\left(1-q^{n}\right)^{24}=q-24 q^{2}+\cdots \in S_{12} \cap \mathbb{Z}[[q]] .
$$

Theorem 1.3. Fix a prime $\ell \geq 5$. Suppose $k=a(\ell-1)+b$ for some $1 \leq a \leq 12$ and $b \in\{4,6,8,10,14\}$ with $b \geq a+2$. If $f(z) \in S_{k} \cap \mathbb{Z}[[q]]$, then $f(z) \mid U(\ell) \equiv 0$ $(\bmod \ell)$.

We prove all three of the above theorems in the next section. We then conclude with a discussion of an analogous function to $\mathcal{P}_{\ell}$ for the small primes $\ell=2,3$ in the final section.

\section{Proof of the theorems}

We begin with some preliminary information about modular forms. Recall that for even $k$ we know $M_{k}$ is a finite-dimensional $\mathbb{C}$-vector space of dimension

$$
d(k):=\operatorname{dim}_{\mathbb{C}}\left(M_{k}\right)=\left\{\begin{array}{lll}
\left\lfloor\frac{k}{12}\right\rfloor+1 & \text { if } k \neq 2 & (\bmod 12), \\
\left\lfloor\frac{k}{12}\right\rfloor & \text { if } k \equiv 2 & (\bmod 12) .
\end{array}\right.
$$

For even $k$ define the function $\delta(k) \in\{0,4,6,8,10,14\}$ by the congruence

$$
\delta(k) \equiv k \quad(\bmod 12)
$$

The above function $\delta(k)$ gives information about the zeroes of $f(z) \in M_{k}$ by the valence formula below. Let $\omega:=e^{2 \pi i / 3}$. If $f(z) \in M_{k}$ and $v_{\tau}(f)$ is the order vanishing of $f$ at $\tau$, then

$$
\frac{k}{12}=v_{\infty}(f)+\frac{1}{2} v_{i}(f)+\frac{1}{3} v_{\omega}(f)+\sum_{\substack{\tau \in \mathcal{H} / \Gamma \\ \tau \notin\{i, \omega\}}} v_{\tau}(f),
$$


where $\mathcal{H}$ denotes the upper half place and $\Gamma=\mathrm{SL}_{2}(\mathbb{Z})$. The valence formula tells us, for example, that $E_{\ell-1}(z) / E_{\delta(\ell-1)}(z)$ and $E_{8}(z) / E_{4}(z)$ are holomorphic modular forms. In addition, we have some well-known facts about the Eisenstein series $E_{k}(z)$. For convenience we define $E_{0}(z):=1 \in M_{0}$.

(1) $E_{k}(z)=1+\cdots \in M_{k} \cap \mathbb{Z}[[q]]$ for each $k \in\{0,4,6,8,10,14\}$.

(2) $E_{\ell-1}(z) \equiv 1(\bmod \ell)$ for $\ell \geq 5$ prime.

We will also need the following theorem of Choie, Kohnen, and Ono CKO05.

Theorem 2.1 (CKO05, Theorem 3.1]). If $f(z) \in M_{k}$ and $g(z) \in M_{12 N}$, then

$$
\text { const }\left(\frac{E_{14-\delta(k)}(z) g(z)}{\Delta(z)^{d(k)+N}} \cdot f(z)\right)=0
$$

where const $(h(z))$ denotes the constant term of the power series $h(z)$.

We are now ready to prove Theorem 1.3 .

Proof of Theorem 1.3. Fix a prime $\ell \geq 5$. Let $k$ be of the specified form. Fix a cusp form $f(z) \in S_{k} \cap \mathbb{Z}[[q]]$. It suffices to show that $a_{f}(\ell n) \equiv 0(\bmod \ell)$ for each $n \geq 1$. This will follow by induction on $n$.

Recall, as $f$ is a cusp form, $a_{f}(0)=0$. Fix an integer $n$ and suppose $a_{f}(\ell m) \equiv 0$ $(\bmod \ell)$ for each $0 \leq m \leq n-1$. Consider the quantity

$$
c(a, b, \ell):=\delta(a(\ell-1))-12(d(b+\delta(a(\ell-1)))-1) .
$$

Define the function

$$
g_{n}(z):=E_{14-b}(z)^{\ell-1} E_{\ell-1}(z)^{b-a-2} E_{4}(z)^{3 \ell(n-1)} \tilde{g}(z),
$$

where

$$
\tilde{g}(z):= \begin{cases}E_{\delta(a(\ell-1))}(z) & \text { if } c(a, b, \ell)>2, \\ E_{14-b} / E_{12-b}(z) & \text { if } c(a, b, \ell)=2, \\ E_{0}(z) & \text { if } c(a, b, \ell)=0, \\ E_{14-b} / E_{16-b}(z) & \text { if } c(a, b, \ell)=-2, \\ 1 / E_{-c(a, b, \ell)}(z) & \text { if } c(a, b, \ell)<-2 .\end{cases}
$$

We first show that the above function $g_{n}(z)$ is a holomorphic modular form by considering each of the three non-trivial possibilities for $c(a, b, \ell)$ separately.

Case i) $c(a, b, \ell)=2$.

$$
g_{n}(z)=E_{14-b}(z)^{\ell-1} E_{\ell-1}(z)^{b-a-2} E_{4}(z)^{3 \ell(n-1)} E_{14-b}(z) / E_{12-b}(z) .
$$

If $c(a, b, \ell)=2$, then $d(b+\delta(a(\ell-1)))=2$ and $\delta(a(\ell-1))=14$. Hence, $\delta(\ell-1)=$ 10. Furthermore, $b \in\{4,6,8\}$. Since $\delta(\ell-1)=10$, it follows by the valence formula that $E_{\ell-1}(z) E_{14-b}(z) / E_{12-b}(z)$ is holomorphic for each $b \in\{4,6,8\}$. It suffices to show that if $c(a, b, \ell)=2$, then $b-a-2>0$. If $b-a-2=0$, then $a$ is even, which contradicts $\delta(a(\ell-1))=14$.

Case ii) $c(a, b, \ell)=-2$.

$$
g_{n}(z)=E_{14-b}(z)^{\ell-1} E_{\ell-1}(z)^{b-a-2} E_{4}(z)^{3 \ell(n-1)} E_{14-b}(z) / E_{16-b}(z) .
$$

If $c(a, b, \ell)=-2$, then $d(b+\delta(a(\ell-1)))=2$ and $\delta(a(\ell-1))=10$, so $\delta(\ell-1)=$ 10 and $b \in\{6,8,10\}$. Again, the valence formula implies that $E_{\ell-1}(z) E_{14-b}(z) /$ $E_{16-b}(z)$ is holomorphic for $b \in\{6,10\}$ if $b-a-2>0$ and for $b=8$ if $b-a-2>1$. If $b-a-2=0$, then $a$ is even, which contradicts the fact that $\delta(a(\ell-1))=10$. If $b=8$ and $b-a-2=1$, then $a=5$, but then $a(\ell-1) \equiv 5(10) \not \equiv 10(\bmod 12)$. 
Case iii) $c(a, b, \ell)<-2$.

$$
g_{n}(z)=E_{14-b}(z)^{\ell-1} E_{\ell-1}(z)^{b-a-2} E_{4}(z)^{3 \ell(n-1)} / E_{-c(a, b, \ell)}(z) .
$$

As $c(a, b, \ell)<-2$, we have $d(b+\delta(a(\ell-1))) \geq 2$. As $b \leq 14$ and $\delta(a(\ell-1)) \leq$ 14 , we have $d(b+\delta(a(\ell-1))) \leq 3$. Moreover, if $\delta(a(\ell-1)) \in\{10,14\}$, then $d(b+\delta(a(\ell-1)))=3$, and if $\delta(a(\ell-1)) \in\{4,6,8\}$, then $d(b+\delta(a(\ell-1)))=2$.

First suppose $\delta(a(\ell-1)) \in\{10,14\}$, so $\delta(\ell-1)=10$. If $\delta(a(\ell-1))=10$, then $-c(a, b, \ell)=14$, and if $\delta(a(\ell-1))=14$, then $-c(a, b, \ell)=10$. By the valence formula, $E_{\ell-1}(z)^{b-a-2} / E_{-c(a, b, \ell)}$ is a holomorphic modular form if $\delta(a(\ell-1))=14$ and $b-a-2>0$ or $\delta(a(\ell-1))=10$ and $b-a-2>1$. As in the previous cases, $\delta(\ell-1)=10$ implies $b-a-2>0$. Suppose $\delta(a(\ell-1))=10$ and $b-a-2=1$. As $d(b+\delta(a(\ell-1)))=3$, this implies that $b=14$. Thus, $a=11$, which yields $11(\ell-1) \equiv 11(10) \not \equiv 10(\bmod 12)$, a contradiction.

Now suppose $\delta(a(\ell-1)) \in\{4,6,8\}$, so $E_{-c(a, b, \ell)}(z) \in\left\{E_{8}(z), E_{6}(z), E_{4}(z)\right\}$, respectively. Begin with $\delta(a(\ell-1))=4$; then $\delta(\ell-1)=4$ or 10 . By the valence formula, $E_{14-b}(z)^{\ell-1} E_{\ell-1}(z)^{b-a-2} / E_{8}(z)$ is a holomorphic modular form if $b-a-$ $2>1$. As $d(b+a(\ell-1))=2$, this implies that $b=8,14$, and so we need only check that $a \neq 5,6,11,12$. Note that in each of these cases $a(\ell-1) \not \equiv 4(\bmod 12)$.

Next suppose $\delta(a(\ell-1))=6$, so $\delta(\ell-1)=6$ or 10 . It again follows by the valence formula that $E_{14-b}(z)^{\ell-1} E_{\ell-1}(z)^{b-a-2} / E_{6}(z)$ is a holomorphic modular form if $b-a-2>0$. If $\delta(a(\ell-1))=6$ and $b-a-2=0$, then $a$ is even, which contradicts $\delta(a(\ell-1))=6$.

Finally, consider $\delta(a(\ell-1))=8$, so $\delta(\ell-1)=4$ or 10 . If $b-a-2>0$ or $b \neq 8,14$, then $E_{14-b}(z)^{\ell-1} E_{\ell-1}(z)^{b-a-2} / E_{4}(z)$ is a holomorphic modular form. If $b=8,14$, then $b-a-2=0$ implies $a=6,12$, for which $\delta(a(\ell-1)) \not \equiv 8(\bmod 12)$.

We can apply Theorem 2.1 to show $a_{f}(\ell n) \equiv 0(\bmod \ell)$. Take $f(z)$ as above and $g(z)=g_{n}(z)$. We have $k=a(\ell-1)-\delta(a(\ell-1))+\delta(a(\ell-1))+b$; it follows that

$$
d(k)=\frac{a(\ell-1)-\delta(a(\ell-1))}{12}+d(b+\delta(a(\ell-1))) .
$$

Similarly,

$$
\begin{aligned}
\delta(k) & =\delta(b+\delta(a(\ell-1))) \\
& =b+\delta(a(\ell-1))-12(d(b+\delta(a(\ell-1)))-1) \\
& =b+c(a, b, \ell)
\end{aligned}
$$

Moreover, $\tilde{g}(z)$ has weight $c(a, b, \ell)$; hence, $g_{n}(z)$ has weight

$$
(14-b)(\ell-1)+(\ell-1)(b-a-2)+12 \ell(n-1)+c(a, b, \ell)=12 N,
$$

where

$$
\begin{aligned}
N & =\ell n-\frac{a(\ell-1)-\delta(a(\ell-1))}{12}-d(b+\delta(a(\ell-1))) \\
& =\ell n-d(k) .
\end{aligned}
$$

Thus, by Theorem 2.1,

$$
\text { const }\left(\frac{E_{14-b-c(a, b, \ell)}(z) g_{n}(z)}{\Delta(z)^{\ell n}} \cdot f(z)\right)=0
$$


Each possibility for $g_{n}(z)$ allows us to conclude that

$$
\text { const }\left(\frac{E_{14-b}(z)^{\ell} E_{\ell-1}(z)^{b-a-2} E_{4}(z)^{3 \ell(n-1)}}{\Delta(z)^{\ell n}} \cdot f(z)\right)=0,
$$

where we use the observation that

$$
E_{14-b-c(a, b, \ell)}(z) \tilde{g}(z)=E_{14-b}(z) .
$$

Now consider the above function modulo $\ell$, recalling $E_{\ell-1}(z) \equiv 1(\bmod \ell)$. The constant term of

$$
\begin{aligned}
& \frac{E_{14-b}(z)^{\ell} E_{\ell-1}(z)^{b-a-2} E_{4}(z)^{3 \ell(n-1)}}{\Delta(z)^{\ell n}} \cdot f(z) \\
& \equiv \frac{E_{14-b}(\ell z) E_{4}(\ell z)^{3(n-1)}}{\Delta(\ell z)^{n}} \cdot f(z) \quad(\bmod \ell) \\
(2.4) & \equiv E_{14-b}(\ell z) E_{4}(\ell z)^{3(n-1)}\left(q^{-\ell}+24+324 q^{\ell}+\cdots\right)^{n} \sum_{n=0}^{\infty} a_{f}(n) q^{n} \quad(\bmod \ell)
\end{aligned}
$$

is congruent to zero modulo $\ell$. Recall that $E_{14-b}(\ell z)$ and $E_{4}(\ell z)^{3(n-1)}$ have leading coefficient 1 , so by multiplying out the above expression, we find for each $0 \leq m \leq$ $n-1$ integers $\alpha_{b, n}(m)$ such that

$$
a_{f}(\ell n)+\sum_{m=0}^{n-1} \alpha_{b, n}(m) a_{f}(\ell m) \equiv 0 \quad(\bmod \ell) .
$$

In particular, $a_{f}(\ell m) \equiv 0(\bmod \ell)$ for each $0 \leq m \leq n-1$ by the inductive hypothesis, and hence $a_{f}(\ell n) \equiv 0(\bmod \ell)$ by induction.

Remark. The above proof fails for the small primes $\ell=2,3$; for example, $E_{\ell-1}(z)$ is not a holomorphic modular form for these primes. We can, however, modify the proof using the fact that $E_{k}(z) \equiv 1(\bmod 2,3)$ for $k \in\{0,4,6,8,10,14\}$. It suffices to show that for each $n \geq 1$ we can find a non-negative integer $N$ such that $d(k)+N=\ell n$, as we can then conclude by Theorem 2.1 that

$$
\text { const }\left(\frac{E_{14-\delta(k)}(z) E_{4}(z)^{3 N}}{\Delta(z)^{d(k)+N}} \cdot f(z)\right) \equiv \operatorname{const}\left(\Delta(\ell z)^{-n} \cdot f(z)\right) \quad(\bmod \ell) .
$$

This yields equations analogous to (2.4) and (2.5). For $\ell=3$ we have $2 a+b \leq 38$, so for each $n \geq 1$ we can take $N=3 n-d(k) \geq 0$, with the exception of $k=36$, where $d(36)=4$. Similarly, for $\ell=2$ we have $a+b \leq 26$, so again we can take $N=$ $2 n-d(k) \geq 0$ with the exception of $k=24$, where $d(24)=3$. For these exceptions the conclusion of Theorem 1.3 does not hold as $\Delta(z)^{\ell}=q^{\ell}+\cdots \in S_{12 \ell} \cap \mathbb{Z}[[q]]$.

We are now ready to prove the main theorem of the paper, which follows as a consequence of Theorem 1.3 above and the following theorem of Ahlgren and Boylan $\mathrm{AB} 03$.

Theorem 2.2 ([AB03, Theorem 3]). If $\ell \geq 5$ is prime and $j \geq 1 \in \mathbb{Z}$, then let $1 \leq \beta_{\ell, j} \leq \ell^{j}$ be the unique integer for which $24 \beta_{\ell, j} \equiv 1\left(\bmod \ell^{j}\right)$. Define $k_{\ell, j}$ by

$$
k_{\ell, j}:= \begin{cases}\frac{\ell^{j-1}(\ell-1)}{2}-\frac{1}{2}\left(\frac{24 \beta_{\ell, j}-1}{\ell^{j}}+1\right) & \text { if } j \text { is odd }, \\ \ell^{j-1}(\ell-1)-\frac{1}{2}\left(\frac{24 \beta_{\ell, j}-1}{\ell^{j}}+1\right) & \text { if } j \text { is even. }\end{cases}
$$


Then there exists a modular form $F_{\ell, j} \in M_{k_{\ell, j}} \cap \mathbb{Z}[[q]]$ such that

$$
\sum_{n=0}^{\infty} p\left(\ell^{j} n+\beta_{\ell, j}\right) q^{n} \equiv \prod_{n=1}^{\infty}\left(1-q^{n}\right)^{\frac{24 \beta_{\ell, j}-1}{\ell j}} \cdot F_{\ell, j}(z) \quad\left(\bmod \ell^{j}\right) .
$$

Proof of Theorem 1.1. We now apply Theorem 2.2 in the case where $\ell$ is any prime at least 5 and $j=1$. In this case

$$
\begin{aligned}
& k_{\ell}:=k_{\ell, 1}=(\ell-1)-12(\lfloor\ell / 24\rfloor+1), \\
& \beta_{\ell}:=\beta_{\ell, 1}=-\frac{\left(\ell^{2}-1\right)}{24}+\ell(\lfloor\ell / 24\rfloor+1)=-\delta_{\ell}+\ell(\lfloor\ell / 24\rfloor+1) .
\end{aligned}
$$

Thus, we have

$$
\sum_{n=0}^{\infty} p\left(\ell n+\beta_{\ell}\right) q^{n} \equiv \prod_{n=1}^{\infty}\left(1-q^{n}\right)^{\frac{24 \beta_{\ell}-1}{\ell}} \cdot F_{\ell, 1} \equiv \prod_{n=1}^{\infty}\left(1-q^{n}\right)^{24(\lfloor\ell / 24\rfloor+1)-\ell} \cdot F_{\ell, 1} \quad(\bmod \ell) .
$$

Multiplication by $q^{\lfloor\ell / 24\rfloor+1} \prod_{n=1}^{\infty}\left(1-q^{n}\right)^{\ell}$ yields

$$
\begin{aligned}
& q^{\lfloor\ell / 24\rfloor+1} \prod_{n=1}^{\infty}\left(1-q^{n}\right)^{\ell} \sum_{n=0}^{\infty} p\left(\ell n+\beta_{\ell}\right) q^{n} \equiv \Delta(z)^{\lfloor\ell / 24\rfloor+1} \cdot F_{\ell, 1} \quad(\bmod \ell), \\
& \prod_{n=1}^{\infty}\left(1-q^{\ell n}\right) \sum_{n=0}^{\infty} p\left(\ell n-\delta_{\ell}\right) q^{n} \equiv \Delta(z)^{\lfloor\ell / 24\rfloor+1} \cdot F_{\ell, 1} \quad(\bmod \ell) .
\end{aligned}
$$

Note that $\Delta(z)^{\lfloor\ell / 24\rfloor+1} \cdot F_{\ell, 1}(\bmod \ell) \in S_{\ell-1} \cap \mathbb{Z}[[q]]$, and so, by Theorem [1.3, we can multiply both sides of the equation above by any Eisenstein series $E_{m}(z)$ for $m \in\{4,6,8,10,14\}$ to find

$$
\left(E_{m}(z) \prod_{n=1}^{\infty}\left(1-q^{\ell n}\right) \sum_{n=0}^{\infty} p\left(\ell n-\delta_{\ell}\right)\right) \mid U(\ell) \equiv 0 \quad(\bmod \ell) .
$$

Applying Euler's Pentagonal Number Theorem, which states

$$
\prod_{n=1}^{\infty}\left(1-q^{\ell n}\right)=\sum_{k \in \mathbb{Z}}(-1)^{k} q^{\ell \omega(k)}
$$

completes the proof.

Equation (2.6) brings us to Theorem 1.2

Proof of Theorem 1.2. Suppose $\ell \geq 5$ is prime. For each $m \in\{4,6,8,10,14\}$ define the numbers $A_{m}(n)$ by

$$
\sum_{n \geq 0} A_{m}(n) q^{n}:=\left(1+b(m) \sum_{n \geq 1} \sigma_{m-1}(n) q^{n}\right)\left(\sum_{k \in \mathbb{Z}}(-1)^{k} q^{\ell \omega(k)}\right)\left(\sum_{n \geq 0} p\left(\ell n-\delta_{\ell}\right) q^{n}\right) .
$$

In particular,

$$
\begin{aligned}
A_{m}(N)=p\left(\ell N-\delta_{\ell}\right) & +\sum_{k \in \mathbb{Z}-\{0\}}(-1)^{k} p\left(\ell(N-\ell \omega(k))-\delta_{\ell}\right) \\
& +b(m) \sum_{\substack{n \geq 1 \\
k \in \mathbb{Z}}} \sigma_{m-1}(n)(-1)^{k} p\left(\ell(N-\ell \omega(k)-n)-\delta_{\ell}\right) .
\end{aligned}
$$


The result now follows by Theorem 1.1 as

$$
\sum_{n \geq 0} A_{m}(n) q^{n} \mid U(\ell) \equiv 0 \quad(\bmod \ell) .
$$

\section{The PRIMES 2 AND 3}

It is difficult to prove anything about the distribution of the partition function $p(n)$ modulo 2 and 3. For these primes $\ell=2,3$, we cannot define $\mathcal{P}_{\ell}(z)$ since $\delta_{\ell}$ is no longer an integer. We can, however, define the following analog for these small primes. For $\ell=2,3$ define

$$
\mathcal{P}_{\ell}^{*}(z):=\sum_{k \in \mathbb{Z}}(-1)^{k} q^{\ell \omega(k)} \sum_{n \geq 0} p(n) q^{n} .
$$

Note that for these small primes

$$
\mathcal{P}_{\ell}^{*}(z) \equiv \prod_{n \geq 0}\left(1-q^{n}\right)^{\ell-1} \quad(\bmod \ell) .
$$

For $\ell=2,3$ define the numbers $a_{\ell}(n)$ by

$$
\sum_{n \geq 0} a_{\ell}(n) q^{n}=\mathcal{P}_{\ell}^{*}(z) .
$$

It follows that

$$
\mathcal{P}_{2}^{*}(z) \equiv \sum_{k \in \mathbb{Z}}(-1)^{k} q^{\omega(k)} \quad(\bmod 2),
$$

and so $a_{2}(N) \equiv 0(\bmod 2)$ precisely when $N$ is not a pentagonal number. Similarly,

$$
\mathcal{P}_{3}^{*}(z) \equiv \sum_{m, n \in \mathbb{Z}}(-1)^{m+n} q^{\omega(m)+\omega(n)} \quad(\bmod 3) .
$$

In particular, $a_{3}(N) \equiv 0(\bmod 3)$ for $N$ that do not satisfy $24 N+2=(6 m+1)^{2}+$ $(6 n+1)^{2}$, where $m, n \in \mathbb{Z}$. Note that while it is difficult to prove anything about $p(n)(\bmod 2,3)$, in sharp contrast we have that $a_{\ell}(n) \equiv 0(\bmod \ell)$ with arithmetic density one.

\section{ACKNOWLEDGEMENTS}

The author thanks Ken Ono for his advising, Jeremy Rouse for assistance with the computer system MAGMA, and the referee for splendid and insightful comments.

\section{REFERENCES}

[AB03] Scott Ahlgren and Matthew Boylan, Arithmetic properties of the partition function, Invent. Math. 153 (2003), no. 3, 487-502. MR2000466 (2004e:11115)

[And98] George E. Andrews, The theory of partitions, Cambridge Mathematical Library, Cambridge University Press, Cambridge, 1998. MR1634067 (99c:11126)

[AO01] Scott Ahlgren and Ken Ono, Addition and counting: the arithmetic of partitions, Notices Amer. Math. Soc. 48 (2001), no. 9, 978-984. MR1854533 (2002e:11136)

[CKO05] Youngju Choie, Winfried Kohnen, and Ken Ono, Linear relations between modular form coefficients and non-ordinary primes, Bull. London Math. Soc. 37 (2005), no. 3, 335341. MR2131386 (2005m:11081)

[Kob93] Neal Koblitz, Introduction to elliptic curves and modular forms, Graduate Texts in Mathematics, vol. 97, Springer-Verlag, New York, 1993. MR1216136 (94a:11078)

Department of Mathematics, University of Wisconsin, Madison, Wisconsin 53706

E-mail address: garthwai@math.wisc.edu 